Pulsed flying spot with the logarithmic parabolas method for the estimation of in-plane thermal diffusivity fields on heterogeneous and anisotropic materials

L. Gaverina, J. C. Batsale, A. Sommier, and C. Pradere

Citation: Journal of Applied Physics 121, 115105 (2017); doi: 10.1063/1.4978919

View online: http://dx.doi.org/10.1063/1.4978919

View Table of Contents: http://aip.scitation.org/toc/jap/121/11

Published by the American Institute of Physics

AIP

Publishing HORIZONS

Small Conferences. BIG Ideas.

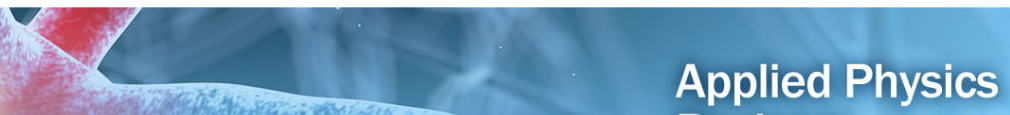

Reviews

SAVE THE DATE!

3D Bioprinting: Physical and Chemical Processes

May 2-3,2017 Winston Salem, NC, USA 


\title{
Pulsed flying spot with the logarithmic parabolas method for the estimation of in-plane thermal diffusivity fields on heterogeneous and anisotropic materials
}

\author{
L. Gaverina, J. C. Batsale, A. Sommier, and C. Pradere ${ }^{a)}$ \\ I2M-TREFLE, UMR CNRS 5295, Esplanade des arts et Métiers 33405 Talence Cedex, France
}

(Received 27 January 2017; accepted 7 March 2017; published online 21 March 2017)

\begin{abstract}
A novel thermal non-destructive technique based on a Pulsed Flying Spot is presented here by considering in-plane logarithmic processing of the relaxing temperature field around the heat source spot. Recent progress made in optical control, lasers, and infrared cameras permits the acquisition of 2D temperature fields and localized thermal excitation on a small area instead of the entire recorded image. This study focuses on a new method based on spatial logarithm analysis of a temperature field to analyse and measure different parameters, such as the in-plane thermal diffusivity and localization of the spot. In this paper, this method is presented and the first results of heterogeneous anisotropic materials are depicted. The in-plane thermal diffusivity is estimated with an error lower than 4\%, and the initial location of the heating spot is determined. Published by AIP Publishing. [http://dx.doi.org/10.1063/1.4978919]
\end{abstract}

\section{INTRODUCTION}

The use of new scanning systems based on a galvanometer mirror allows the easy control of the spatial and temporal displacements of a laser hot spot over a plane surface. Such systems are then suitable for use in developing new flying spot methods as alternatives to the initial flying spot technique, ${ }^{1-4}$ which is based on a constant velocity of the spot. By contrast, instead of the single temperature measurement used in Ref. 5, infrared (IR) thermography allows the transient temperature field measurement and direct processing of a small area around the heating spot. Even if a great number of pulsed spots are deposited, the preliminary step will consist of analysing the effect of a unique spot. To implement such processing, an analysis of the transient field of the temperature response from the analytical expression has been proposed. This method is very close and complementary to the Thermographic Signal Reconstruction (TSR) method used in the case of the 1D flash experiment. ${ }^{6}$ In fact, this technique used the time logarithm of the temperature field, whereas the method proposed here is based on the spatial logarithmic processing of the temperature response. Other methods exist for in-plane thermal diffusivity estimation by using the Fourier transform of the temperature field ${ }^{7-9}$ or by considering the transient evolution of the Gaussian temperature distribution. ${ }^{10,11}$ One drawback of these methods is the number of pixels needed to perform inverse processing. The main goal of this work is based on laser local heating (the heat spot size is lower than the pixel size), and the idea is to propose a method in which only a few pixels around the heating spot are necessary to retrieve the thermal properties.

In this paper, some theoretical aspects are considered. A new estimation method is then presented, and finally, experimental data and results are shown to validate the capability

\footnotetext{
a) Author to whom correspondence should be addressed. Electronic mail: c.pradere@i2m.u-bordeaux1.fr
}

of the method by estimating the in-plane diffusivities, position of the spot, and nature of the in-plane heat transfer in a heterogeneous and anisotropic sample.

\section{MATERIAL AND EXPERIMENTAL METHODS}

\section{A. Setup}

The experimental setup in Figure 1 is composed of a laser diode (wavelength $976 \mathrm{~nm}$ ) of $330 \mathrm{~mW}$ power. To collimate the laser beam, an optical collimator system (Thorlabs) is used. A Dual-Axis Scanning Galvo System (Thorlabs GVS112/M) was used to control the spatial displacement of the laser spot. The principle of the laser beam deviation towards the sample is shown in Figure 1. To focus the laser beam on the surface, an f-theta scan lens was used with a focusing length of $160 \mathrm{~mm}$. With this focal length, the scanning area is equal to a square of $11 \mathrm{~cm} \times 11 \mathrm{~cm}$, and the resulting diameter of the focused spot is $26 \mu \mathrm{m}$ with a minimum displacement of $4.5 \mu \mathrm{m}$. In practice, the angular positions of the galvanometric system are related to a couple of coordinates expressed as voltage (V). Indeed, the range of angular positions included within $-20^{\circ}$ and $20^{\circ}$ is discretized in terms of voltage applied to the motor in the corresponding range of $-10 \mathrm{~V}$ to $10 \mathrm{~V}$. Thus, the angular position calibration, applied voltage, and resulting displacement are set according to the following relationship: $\Delta D_{f}=2 f \tan (\Delta \theta)$ $=2 f \tan \left(\frac{\Delta V}{2}\right)$.

This laser diode is mounted horizontally, and the beam is reflected with a dichroic mirror (MD, treated to reflect $95 \%$ of the visible light from $700 \mathrm{~nm}$ to $1000 \mathrm{~nm}$ and to transmit $95 \%$ of the infrared radiation between 2 and $16 \mu \mathrm{m}$ ). To measure the temperature fields, an IR camera MCT (FLIR SC7000, $320 \times 256$ pixels, pitch $30 \mu \mathrm{m}$, and spectral band from 7 to $14 \mu \mathrm{m}$ ) was used with an infrared objective lens (focal $25 \mathrm{~mm}$ ). Finally, the resulting spatial resolution is approximately $250 \mu \mathrm{m}$ per pixel. 


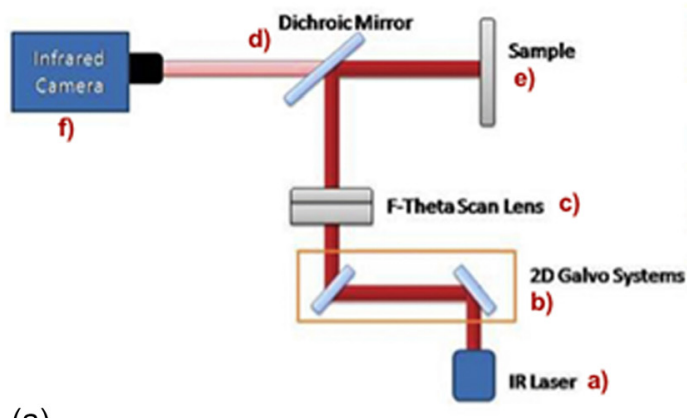

(a)

\section{B. Material}

In this part, several measurements will be performed on the sample illustrated in Figure 3.

This sample was manually assembled to realize a heterogeneous anisotropic material. It is composed of several homogeneous and anisotropic materials. The first sub-sample in Figure 2(a) is a composite material of carbon/epoxy elaborated with $2 \mathrm{D}$ fibre tissues. The second sub-sample in Figure 2(b) is a homogeneous and isotropic Plexiglass plate. The third one in Figure 2(c) is a pin wood heterogeneous and anisotropic sample with cellulose fibres oriented along the $\mathrm{x}$ direction, and the last one in Figure 2(d) is another carbon/ epoxy elaborated with fibres oriented along the $y$ direction. The total dimensions of the sample are $5 \mathrm{~cm} \times 5 \mathrm{~cm}$. To

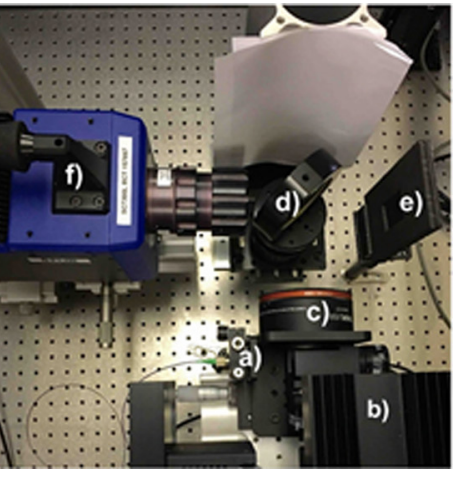

FIG. 1. Experimental setup: A scheme of the system and its photography.

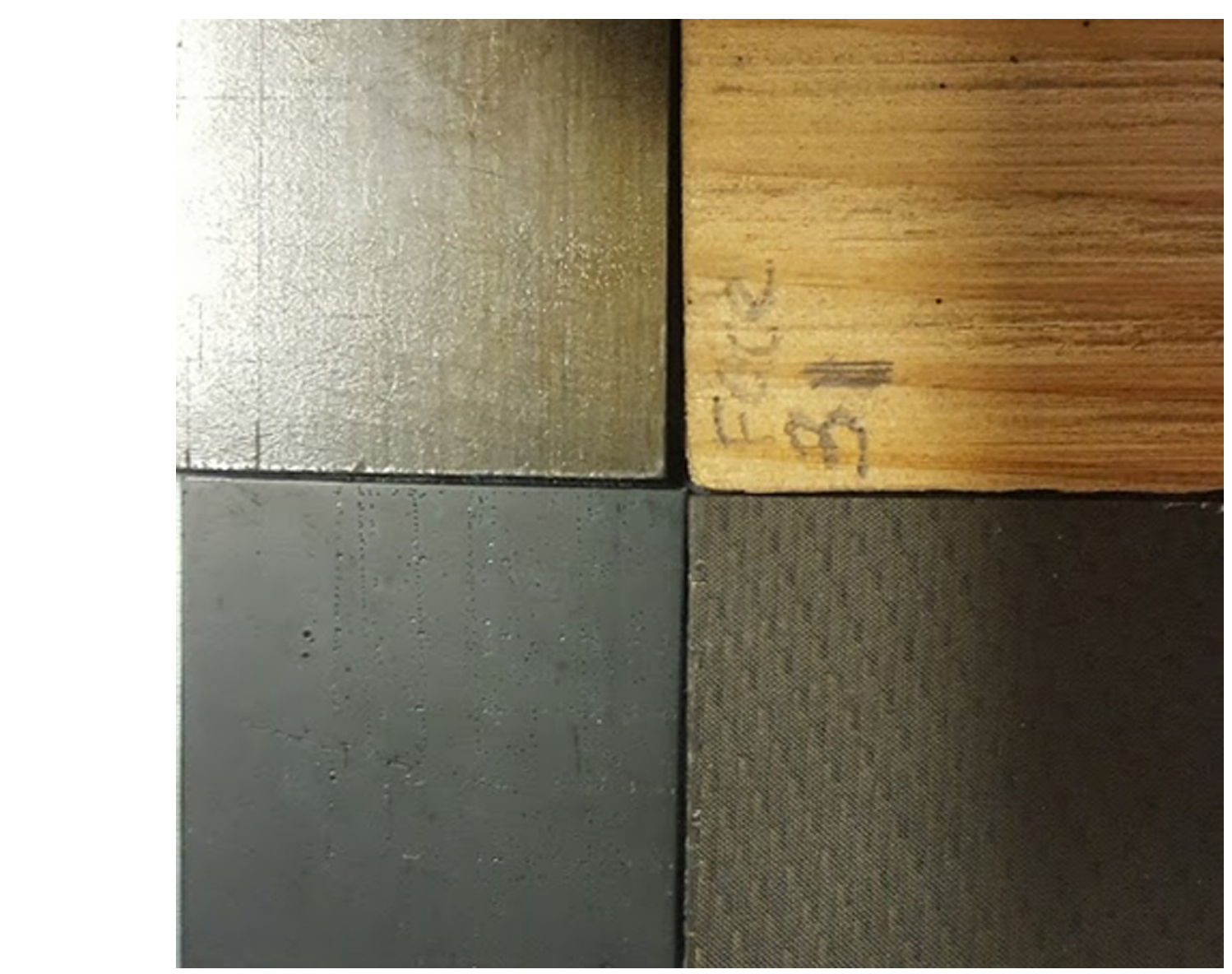

FIG. 2. Heterogeneous sample manually assembled: (a) isotropic carbon/epoxy composite material with fibre tissue, (b) homogeneous and isotropic Plexiglas, (c) pin wood heterogeneous sample with fibres oriented along the $\mathrm{x}$ direction, and (d) anisotropic carbon/epoxy composite material with fibres oriented along

the y direction.

evidence the ability of the proposed method to also detect cracks or interface defects, a space was added between all the sub-samples. The thickness of each sample is not the same, but in this study where the experimentation is performed in the front face, it has no influence on the modellisation of the proposed method and the experimental results.

\section{Pulsed flying spot method}

Due to the high versatility of the galvanometric system, infinite shapes could be generated for the laser displacement. In this paper addressing the Pulse Flying Spot technique, the most basic shape corresponding to a Cartesian laser grid is applied. With this assumption, the main parameters illustrated in Figure 2 are (i) the definition of the area to be 
scanned, (ii) the number of positions according to the directions of $x$ and/or $y$, (iii) the temporal form of thermal excitation to a given position, (iv) the frequency of movement between 2 positions, and (v) the number of cycles or repetitions of phases (ii) to (iv). From these global parameters, only 3 could be considered as important in regard to the thermal excitation: the number of spots (or request) according to the $\mathrm{x}$ and $\mathrm{y}$ directions, which leads to a matrix grid of $N x$ and $N y$ excitations, the duration $\tau$ of the pulse to a given position, and the time interval between 2 positions. As a consequence, the formulation of the front face boundary condition of a Grid Pulsed Flying Spot laser excitation can be expressed as follows:

$$
\begin{aligned}
Q(x, y, t)= & \sum_{k=1}^{N(I * J)}\left(\sum_{j=1}^{J}\left(\sum_{i=1}^{I} \delta(x-i \Delta x)\right) \delta(y-j \Delta y)\right) \\
& \times \delta(t-k \Delta t) .
\end{aligned}
$$

In this study, the measure will be to achieve the $7 \times 7$ impacts on the overall sample for different successive spatial shifts according to $\mathrm{x}$ or $\mathrm{y}$. An illustration of the field of temperature obtained after a pass is shown in Figure 4. The interest seeks the entire surface of the sample and makes several passes. Thus, 4 total passages will be conducted for approximately 200 estimates of in-plane thermal diffusivities. The parameters of the thermal excitation are (i) duration of the pulse equal to $50 \mathrm{~ms}$, (ii) the laser focused beam diameter $\mathrm{d}=26 \mu \mathrm{m}$, and (iii) the power of the laser source of 330 $\mathrm{mW}$. The parameters for the acquisition with the IR camera of the temperature fields in Figure 7 are as follows: (i) the spatial resolution of one pixel of the infrared camera is fixed at $250 \mu \mathrm{m}$ (with an uncertainty of $\pm 4 \mu \mathrm{m}$ ) per pixel, (ii) the frequency of acquisition of the camera is fixed at $200 \mathrm{~Hz}$, (iii) the integration time is equal to $500 \mu \mathrm{s}$, and (iv) the acquisition is completed over a period of $t=7 \mathrm{~s}$, which represents 1500 images.

As a first step, it is interesting to note the quality of the measures with low noise levels. An example of temperature measured at different times is presented in Figure 4. The field in Figure 4(c) during the thermal relaxation can already reveal the highly heterogeneous nature of this material.
Indeed, it is possible to observe that the part related to the Plexiglas (b) presents a higher temperature with rather circular isotherm characteristics of a homogeneous and isotropic sample. However, the parts related to anisotropic materials clearly highlight coverage, depending on the y direction for the material (Figure 2(d)) so that it is much more pronounced according to the $\mathrm{x}$ direction for the (Figure 2(c)) sample of maritime pine. Similarly, the borders separating the different sets are clearly distinguishable in this figure. Therefore, the goal of this PFS method is highlighted. Finally, in Figure 4(d), the temporal evolution of the pulsed response of each impact is presented for the different materials.

\section{THERMAL MODELLISATION AND INVERSE PROCESSING}

\section{A. The direct problem}

The measurements performed in Figure 4(d) are identical to a pulsed source spatially distributed along a Cartesian grid and sequentially time shifted. The thermal modellisation of such a problem could then be analytically solved by the response to a Dirac pulse.

The front face analytical expression of the temperature response field of a pulsed source, imposed on the front face $(\mathrm{z}=0)$ of a homogeneous and anisotropic semi-infinite medium, is written as follows: $:^{10,12}$

$$
\begin{aligned}
T^{*}(x, y, z=0, t)= & \frac{Q}{\rho c_{p}} \frac{\exp \left(-\frac{\left(x-x_{0}\right)^{2}}{4 a_{x} t}\right)}{\sqrt{\pi a_{x} t}} \\
& \times \frac{\exp \left(-\frac{\left(y-y_{0}\right)^{2}}{4 a_{y} t}\right)}{\sqrt{\pi a_{y} t}} \frac{1}{\sqrt{\pi a_{z} t}},
\end{aligned}
$$

where $x_{0}$ and $y_{0}$ are the spatial coordinates of the laser spot versus $x$ and $y$ directions $(\mathrm{m}) ; a_{\mathrm{x}}, a_{\mathrm{y}}$, and $a_{\mathrm{z}}$ represent the thermal diffusivities versus the $x, y$, and $z$ directions $\left(\mathrm{m}^{2} \mathrm{~s}^{-1}\right) ; Q$ is the energy of the pulse point source $(\mathrm{J})$, and $\rho c$ corresponds to the heat capacity per unit volume $\left(\mathrm{J} \mathrm{m}^{-3} \mathrm{~K}^{-1}\right)$. Due to the separability properties, Equation (2) is divided by the spatial

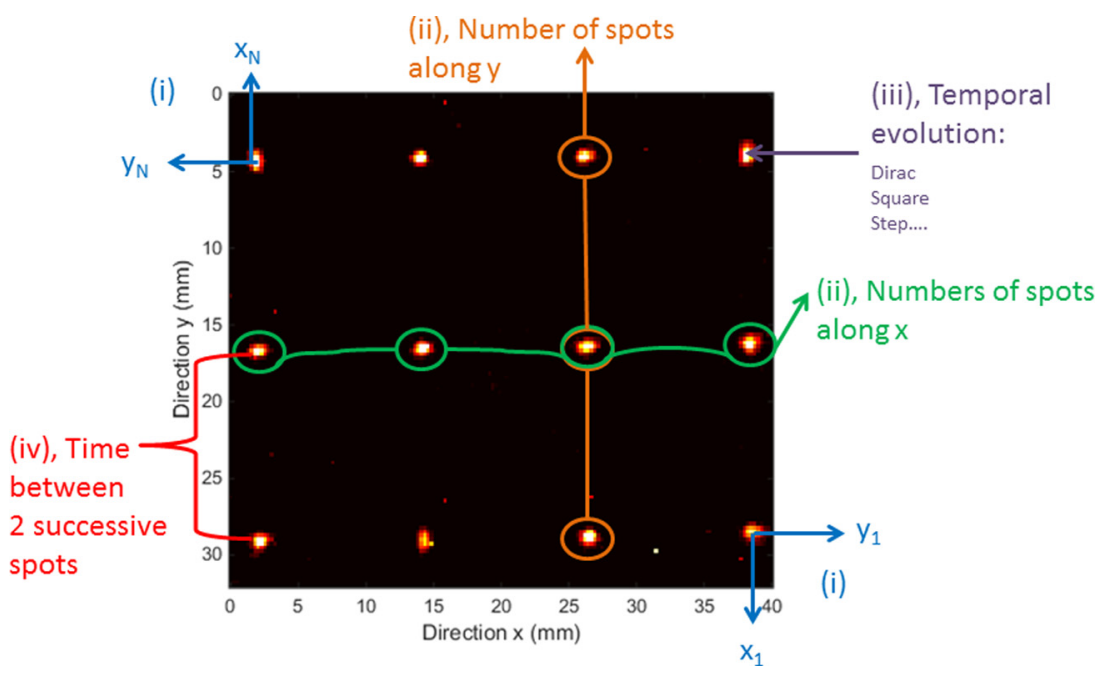

FIG. 3. Schema of the key point parameters to realize the Grid Pulsed Flying Spot. 


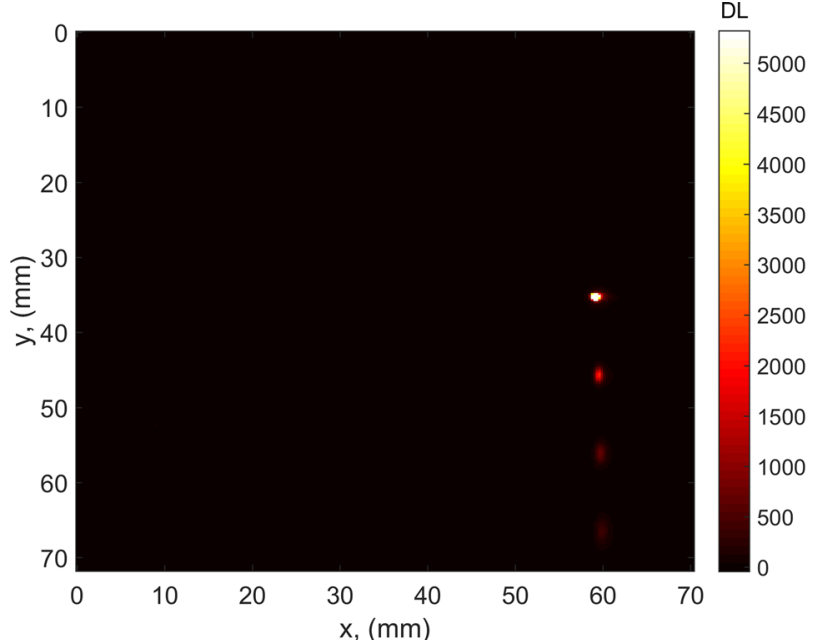

(a)

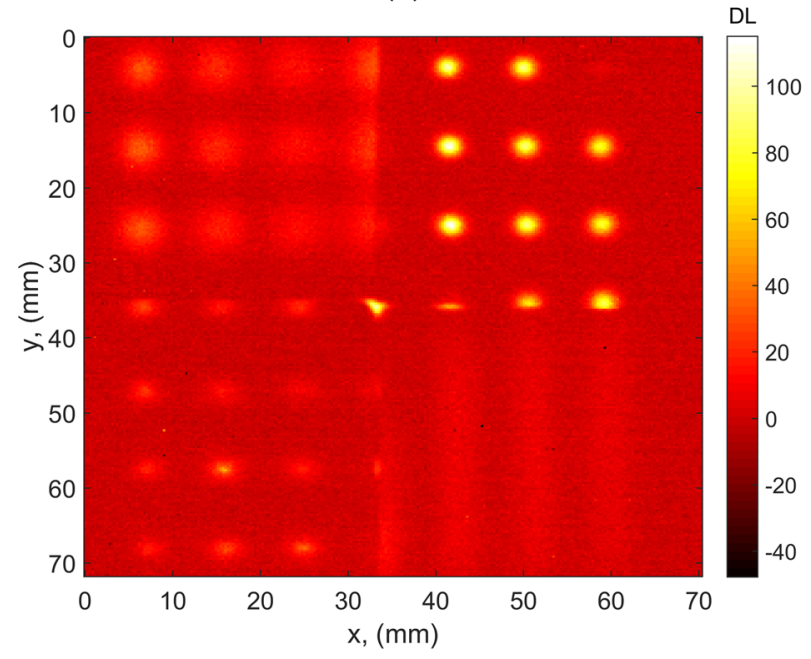

(c)

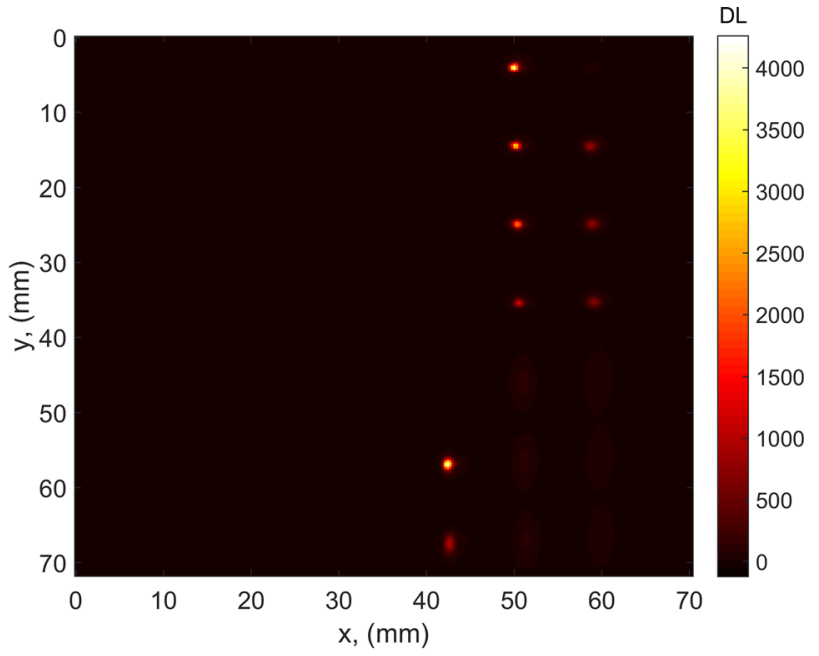

(b)

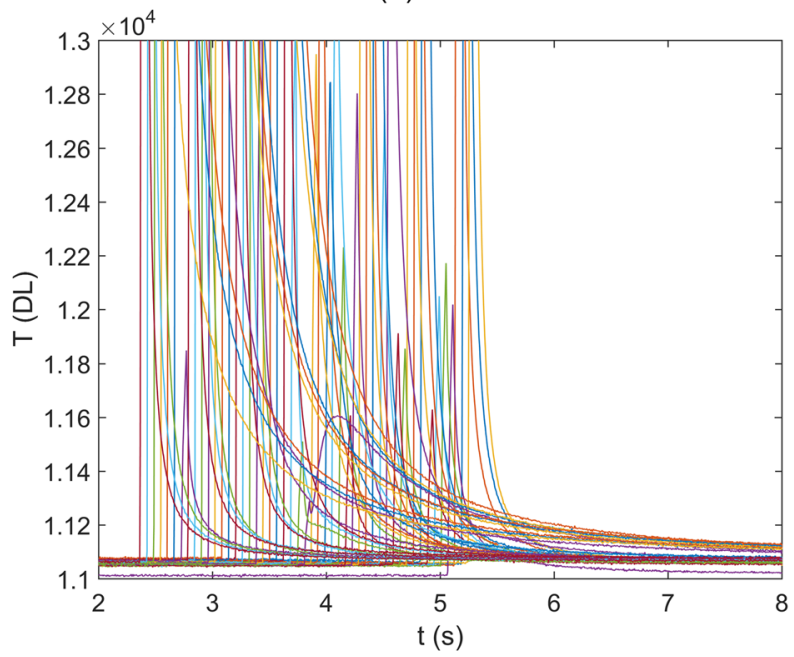

(d)

FIG. 4. Pulsed Grid Flying Spot: (a) Temperature fields after 4 spots, (b) temperature after 16 spots, (c) temperature fields during the relaxation after the 49 spots, and (d) temperature at the location of each spot as a function of time.

averaged temperature to obtain the following $2 \mathrm{D}$ and transient thermal response:

$$
\begin{aligned}
T(x, y, t) & =\frac{T^{*}(x, y, z=0, t)}{\langle T(x, y, z=0, t)\rangle_{x, y}} \\
T(x, y, t) & =\frac{\exp \left(-\frac{\left(x-x_{0}\right)^{2}}{4 a_{x} t}\right)}{\sqrt{\pi a_{x} t}} \frac{\exp \left(-\frac{\left(y-y_{0}\right)^{2}}{4 a_{y} t}\right)}{\sqrt{\pi a_{y} t}},
\end{aligned}
$$

where $\langle T(x, y, z=0, t)\rangle_{x, y}=\frac{Q}{\rho c_{p}} \frac{1}{\sqrt{\pi a_{z}} t^{\circ}}$

\section{B. The logarithmic parabolic method}

If the space average following the $\mathrm{x}$ and $\mathrm{y}$ directions is considered, it yields a 1D transient temperature distribution that is given by

$$
\left\{\begin{array}{l}
\langle T(x, y, t)\rangle_{x}=\frac{1}{l} \int_{0}^{l} T(x, y, z=0, t) d x \\
\langle T(x, y, t)\rangle_{y}=\frac{1}{L} \int_{0}^{L} T(x, y, z=0, t) d y
\end{array}\right.
$$

where $l(\mathrm{~m})$ and $L(\mathrm{~m})$ are the dimensions of the rectangular domain in the $x$ and $y$ directions, respectively. The consideration of the spatial logarithm of the previous expression then yields the following expression:

$$
\left\{\begin{array}{l}
\ln \left(\langle T(x, y, t)\rangle_{x}\right) \approx \ln \left(\frac{k}{t^{\alpha}}\right)-\frac{\left(y-y_{0}\right)^{2}}{4 a_{y} t} \\
\ln \left(\langle T(x, y, t)\rangle_{y}\right) \approx \ln \left(\frac{k}{t^{\alpha}}\right)-\frac{\left(x-x_{0}\right)^{2}}{4 a_{x} t}
\end{array}\right.
$$

where $\alpha=-\frac{1}{2}$ for a semi-infinite medium and thin layer versus the $z$ direction and $k$ is a known constant. The expression can be assimilated to the following polynomial relation:

$$
\left\{\begin{array}{l}
\ln \left(\langle T(x, y, t)\rangle_{x}\right)=\beta_{0}(t)+\beta_{1}(t) y+\beta_{2}(t) y^{2} \\
\ln \left(\langle T(x, y, t)\rangle_{y}\right)=\beta_{0}(t)+\beta_{1}(t) x+\beta_{2}(t) x^{2}
\end{array}\right.
$$




$$
\left\{\begin{array}{l}
\beta_{2}(t)=-\frac{1}{4 a_{x} t} \text { or }-\frac{1}{4 a_{y} t} \\
\beta_{1}(t)=\frac{x_{0}}{2 a_{x} t} \text { or } \frac{y_{0}}{2 a_{y} t} \text { or }-\frac{1}{2 \beta_{2}(t)}=\frac{x_{0}}{\beta_{1}(t)} \text { or }-\frac{1}{2 \beta_{2}(t)}=\frac{y_{0}}{\beta_{1}(t)} . \\
\beta_{0}(t)=-\frac{x_{0}^{2}}{4 a_{x} t}+\ln \left(\frac{k}{t^{\alpha}}\right) \text { or }-\frac{y_{0}^{2}}{4 a_{y} t}+\ln \left(\frac{k}{t^{\alpha}}\right)
\end{array}\right.
$$

Several comments arise from the previous expressions:

- $\beta_{2}(t)$ is dependent only on the thermal diffusivities $a_{\mathrm{x}}$ and $a_{\mathrm{y}}$. It is thus a suitable way to verify the in-plane homogenous transfer at different time steps, independent of the transverse heat transfer and the position of the spot.

- When the in-plane thermal diffusivities were estimated using $\beta_{2}(t)$, the estimation of $x_{0}$ and $y_{0}$ could be conducted with the $\beta_{1}(t)$ parameter or with the direct relation between $\beta_{1}(t)$ and $\beta_{2}(t)$.

- The estimation of $\beta_{0}(t)$ is directly related to a $1 \mathrm{D}$ front face flash experiment; this type of signal contributes to the process. For example, the TSR method ${ }^{6}$ could be used to

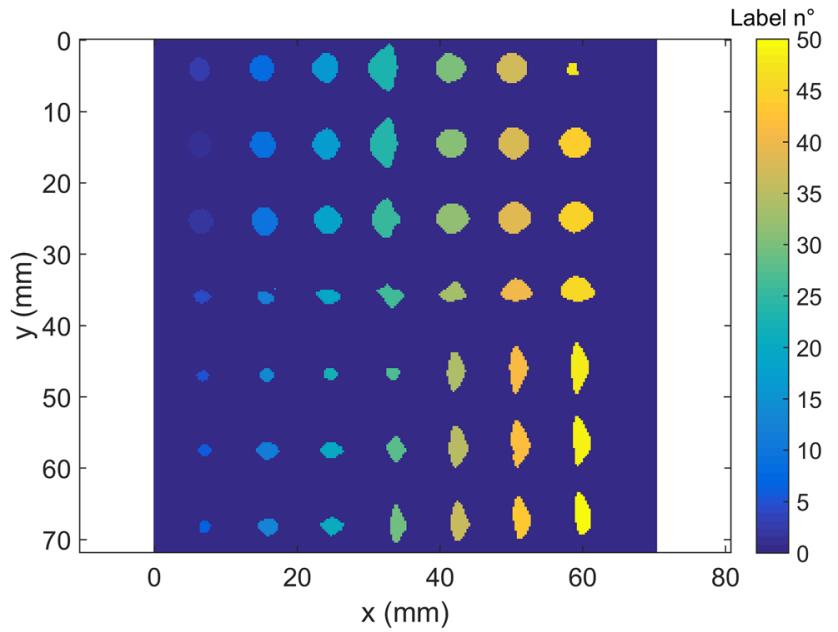

(a)

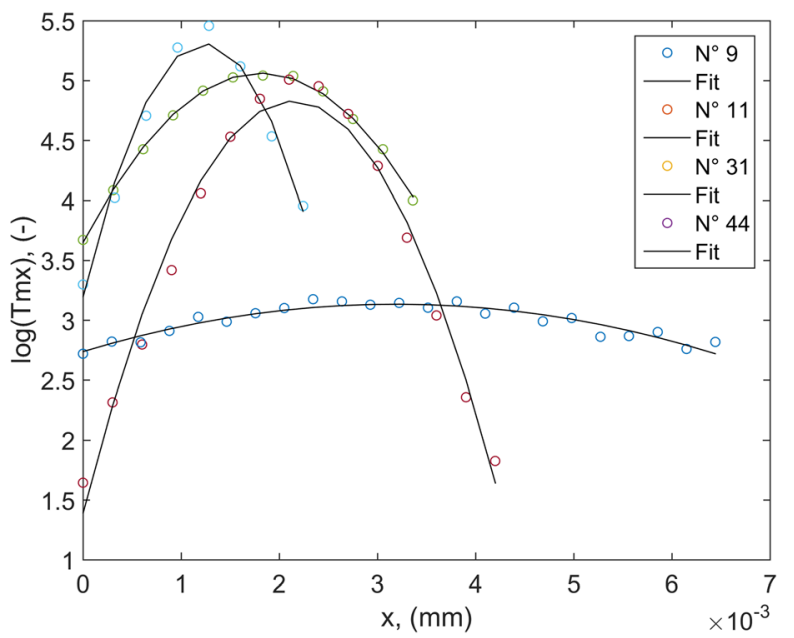

(c) estimate the parameter on an $\ln (T)$ versus $\ln (t)$ graph representation.

\section{The inverse processing}

For convenience, the measurement noise is considered uniform on the distribution and expressed as follows:

$$
\widehat{T}\left(x, y, t_{i}\right)=T\left(x, y, t_{i}\right)+e_{T\left(x, y, t_{i}\right)},
$$

where $e_{T\left(x, y, t_{i}\right)}$ is the random fluctuation added to a signal $T\left(x, y, t_{i}\right)$. It is considered to have a zero mean and uniform standard deviation (the covariance matrix is diagonal).

The space average of Equation (7) is given by

$$
\bar{T}_{\xi}=\bar{T}_{\xi}+e_{\bar{T}_{\xi}},
$$

where $\xi$ represents the $\mathrm{x}$ and $\mathrm{y}$ directions. From the measurements, a polynomial fitting can be implemented at each time $t$ (Figures 5(b) and 5(d)). Parameter $\hat{\beta}_{n}$ can be estimated by using a linear least-squares relationship. It can be expressed

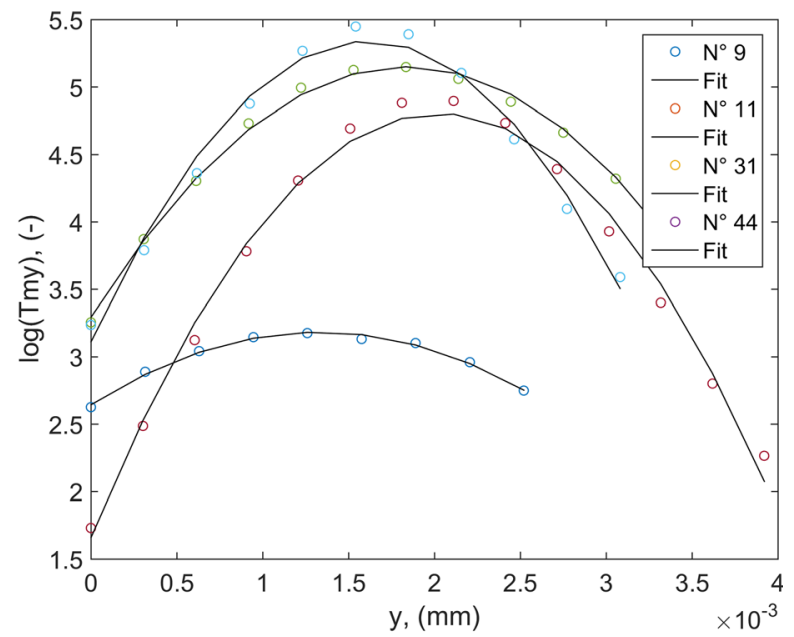

(b)

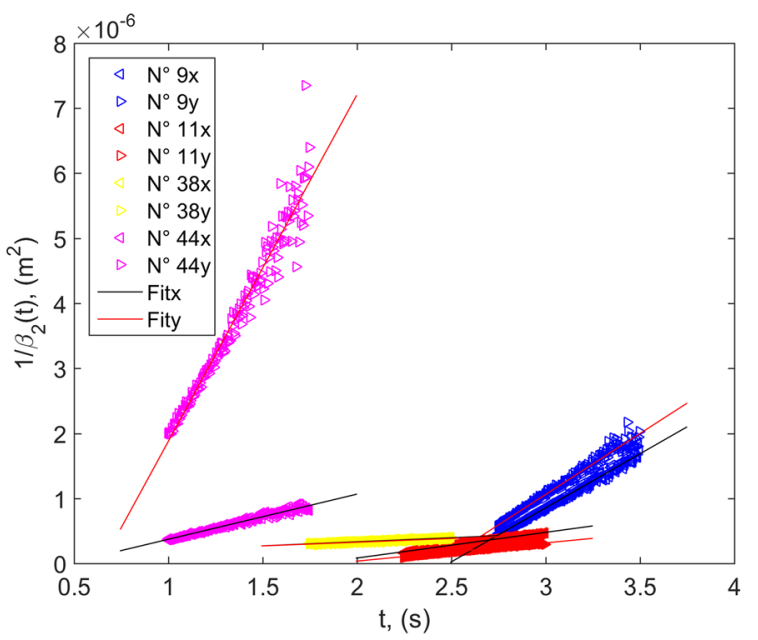

(d)

FIG. 5. Estimation of the thermal properties in the plane through the method of the parabolas: (a) labelling of the laser sources following the grid space, (b) logarithmic representation of the average temperature along the $\mathrm{x}$ direction and the resulting fit for each material, (c) logarithmic representation of the average temperature along the y direction and the resulting fit for each material, and (d) in-plane estimated thermal diffusivities by linear regression from the coefficients obtained using the LPM (b) and (c). 
as a linear combination of the logarithm of the measured temperature $\ln \left(\bar{T}_{\xi}\right)$ with a parameter such that $\ln \left(\bar{T}_{\xi}\right)$ $=S \cdot \hat{\beta}$, where $S$ is the sensitivity matrix.

$$
\left[\begin{array}{c}
\ln \left(\bar{T}_{\xi 1}\right) \\
\vdots \\
\ln \left(\bar{T}_{\xi_{N}}\right)
\end{array}\right]=\left[\begin{array}{ccc}
1 & \xi_{1} & \xi_{1}^{2} \\
\vdots & \vdots & \vdots \\
1 & \xi_{N} & \xi_{N}^{2}
\end{array}\right] .
$$

The method of least squares assumes that there is constant variance in the noise, but in this case, the method of weighted least squares (Equation (11)) must be used because the ordinary least squares assumption of constant variance in the noise is violated (heteroscedasticity), as shown in Equation (10)

$$
\ln \left(\bar{T}_{\xi}\right)=\ln \left(\bar{T}_{\xi}\right)+\frac{e_{\bar{T}_{\xi}}}{\bar{T}_{\xi}} .
$$

The optimal estimation then yields:

$$
\hat{\beta}=\left(S^{T} W S\right)^{-1} S^{T} W \ln \left(\bar{T}_{\xi}\right),
$$

where $\mathrm{W}$ is given by the diagonal elements of the variancecovariance matrix in the noise.

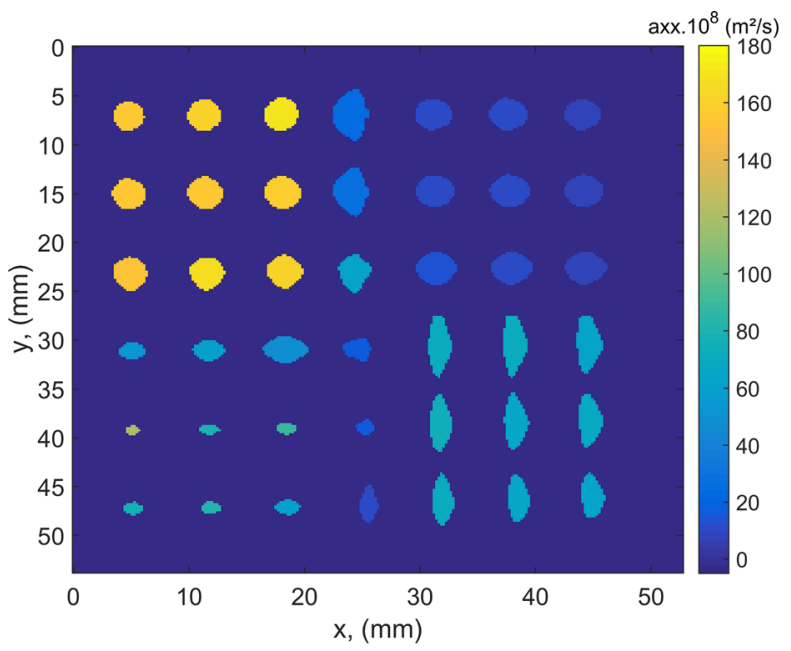

(a)

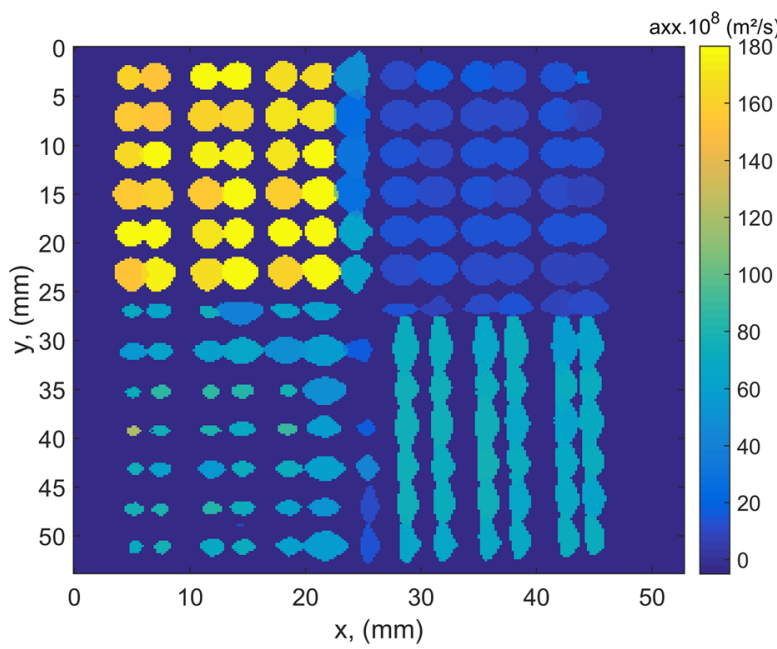

(c)

\section{EXPERIMENTAL RESULTS AND DISCUSSION OF HETEROGENEOUS AND ANISOTROPIC MATERIALS}

The results presented in this section come from the set of experiments illustrated in Figure 4 and based on the GPFS technique described in Section II associated with the LPM. From the previous theoretical considerations, it is necessary to validate the different steps of the parameter estimation shown in Equation (6). First, as known by IR thermography, the offset due to the surroundings is subtracted from all the acquired images. From one set of experiments (as shown in Figure 2), a threshold method ${ }^{13-15}$ is used to mark the centre of each laser spot, as shown in Figure 5(a).

From this location, the two marginal averages of Equation (4) are calculated as functions of time. To optimize the area where the parabolic fit of Equation (6) is performed, the spatial first-order derivative of the marginal averages is calculated for both the directions. The detection of the maximum and the minimum of this derivative allows the optimal zone for the parabolic fitting to be defined. Inside this optimal area, quadratic fits (Equation (12)) are realized for the several sub-materials and illustrated in Figures 5(b) and 5(c). From these fits, the 3 coefficients of the polynomial of order

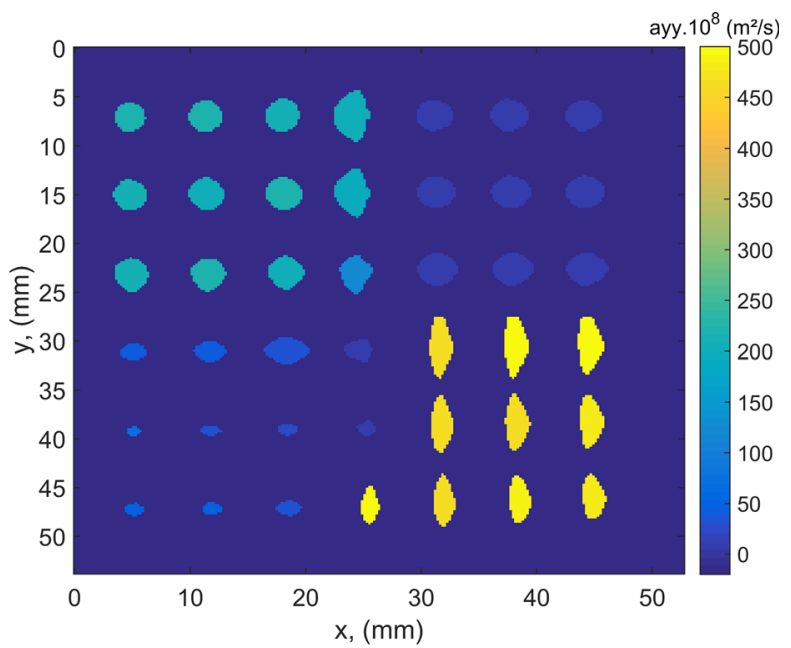

(b)

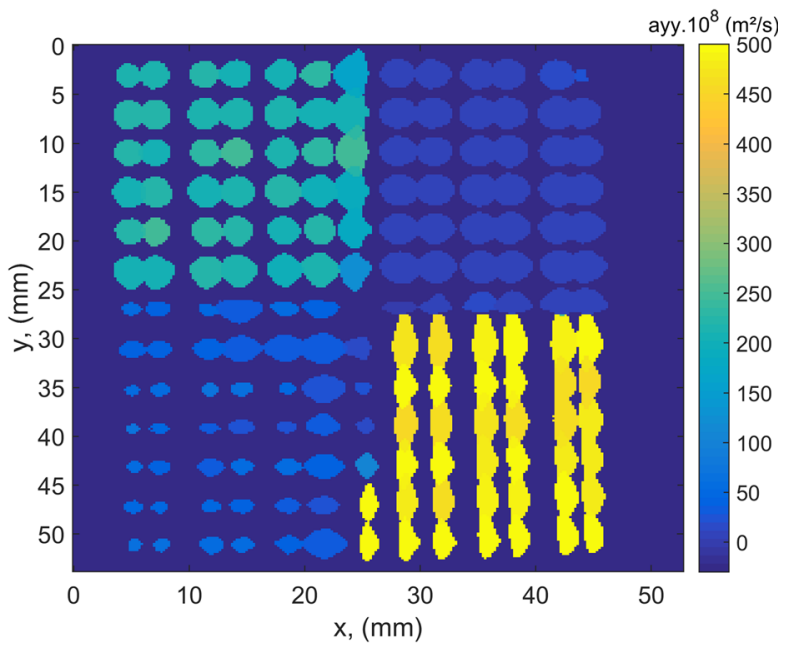

(d)

FIG. 6. Estimation of the in-plane thermal diffusivity fields by using LPM: (a) first grid of the laser path estimated along the $\mathrm{x}$ direction, (b) first grid of the laser path estimated along the $\mathrm{y}$ direction, (c) estimation along $\mathrm{x}$ directions after a set of 4 passages, and (d) the same along the $\mathrm{y}$ direction. 


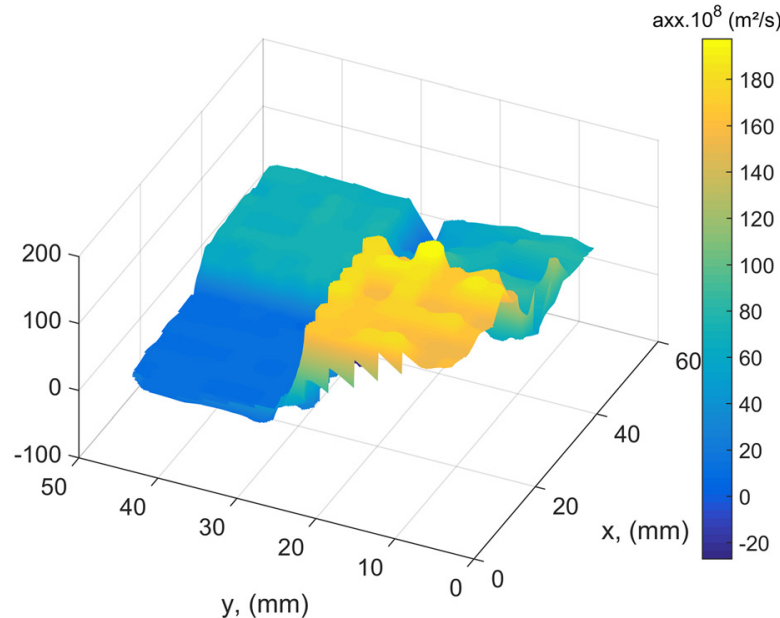

(a)

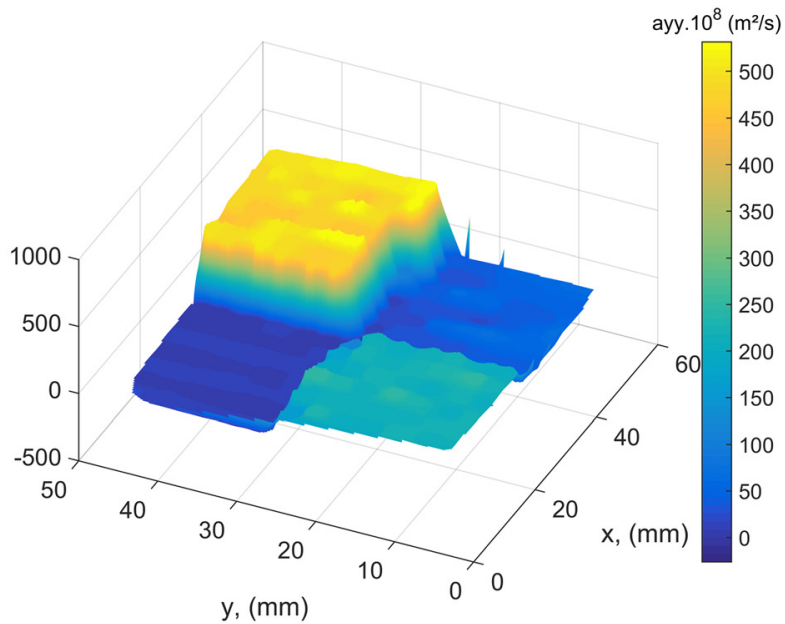

(b)

FIG. 7. In-plane thermal diffusivity fields of heterogeneous and anisotropic materials estimated using the LPM with PFS thermal excitation: (a) diffusivities along the $\mathrm{x}$ direction and (b) along the $y$ direction.

2 are estimated for each time step and for each of the different impacts of the laser source. By using the linear fit from Equation (7), the thermal diffusivities and the location of the laser spot can be retrieved (see Figure 5(d)). These slopes according to Equation (7) are directly proportional to the thermal diffusivities. To avoid errors in estimating the thermal diffusivity and the laser location, the best estimation corresponds to the thermal relaxation, where the linear behaviour is observed with a high signal-to-noise ratio. Over a short time, the estimation is biased because the specimen is illuminated by a Gaussian laser beam of radius $R .^{10,11,17,18}$ Thus, the y-intercept of this curve is proportional to the radius of the beam laser instead of being equal to zero as predicted by using the model Equation (2) of a Dirac spatial pulse.

First, to validate the measured data in the case of homogeneous isotropic and anisotropic samples, particular attention is paid to the results of the estimation of sub-samples $b$ and d (see Figure 4) for which the data are given in the literature. ${ }^{16,19}$ The estimated in-plane thermal diffusivities (Figure $5(\mathrm{~d})$ ) of the Plexiglass sample (Figure 4(b)) are $a_{\mathrm{x}}=1.1$ $\times 10^{-7} \mathrm{~m}^{2} \mathrm{~s}^{-1}$ and $a_{\mathrm{y}}=1.13 \times 10^{-7} \mathrm{~m}^{2} \mathrm{~s}^{-1}$. These values are in good agreement with $a=1.09 \times 10^{-7} \mathrm{~m}^{2} \mathrm{~s}^{-1}$ given by Ref. 16 with a difference of less than $2 \%$. Moreover, the very low difference between $a_{x}$ and $a_{y}$ confirms the isotropy of the sample. Concerning the sample of carbon/epoxy (Figure 4(d)), the estimated values by using Equation (6) of the in-plane thermal diffusivities (Figure 5(d)) are $a_{\mathrm{x}}$ $=6.74 \times 10^{-7} \mathrm{~m}^{2} \mathrm{~s}^{-1}$ and $a_{\mathrm{y}}=5.44 \times 10^{-6} \mathrm{~m}^{2} \mathrm{~s}^{-1}$. These values confirm the anisotropic assumption of the sample with a calculated ratio of $a_{\mathrm{y}}$ to $a_{\mathrm{x}}$ of 8 . This behaviour is in very good agreement with the values of the thermal diffusivity found in the literature ${ }^{19}$ with respective differences of $1.92 \%$ in the $\mathrm{x}$ direction and $3.45 \%$ in the $\mathrm{y}$ direction. These results allow us to validate the method to realize the complete thermal characterization of the heterogeneous sample in Figure 4. For that, this procedure is illustrated in Figures 6(a) and 6(b) in the case of a single path and then generalized to Figures 6(c) and 6(d) for 4 paths. Here, it should be noted that after 4 successive paths, each material has been estimated approximately 36 times on its entire surface.

These results clearly show the relevance of the tool (methods and measures) developed in this work. In addition, the chosen example (heterogeneous anisotropic material) also highlights the ability of this approach to quantitatively characterize a field of thermal diffusivities in the plan. It should be noted that the anisotropic character is well found and identified for sub-materials a, c, and d. Finally, "cracks" that voluntarily left spaces during the assembly of these 4 under-materials are also identified. Finally, from this set of measures, it is possible to refine the mesh or grid of scanning to fill the missing estimations. In this case, we assumed the number of paths to be sufficient and thus achieve a linear data interpolation to obtain full property fields presented in Figure 7.

In conclusion to this part, the efficiency and simplicity of all the measurement devices and methods of parabolas for the characterization of heterogeneous anisotropic materials should be noted. Indeed, the property field results validate the ability of this tool. It should be noted that in this very quantitative case, successive scans are quite long, on the order of $7 \mathrm{~s}$.

\section{CONCLUSION}

The results presented in this paper concern the preliminary step to develop a new flying spot method. Here, the case of a pulsed grid flying spot was broached. The proposed LPM data processing method can be considered to be an extension of the Shepard method because the estimation is processed by considering the spatial logarithmic expression of the temperature field. By using this spatial logarithmic method, it becomes possible to estimate both the in-plane thermal diffusivities and the initial location of the heating spot. The robustness and simplicity of the proposed method were validated by two examples: one on a homogeneous and isotropic polymer sample and the other on a homogeneous and anisotropic carbon/epoxy sample. For both the cases, the 
retrieved results of the in-plane thermal diffusivities are in very good agreement with those of the literature (error lower than $4 \%$ ). The entire processing was then applied to a heterogeneous and anisotropic sample. The obtained results show the ability to obtain full fields of in-plane thermal properties. Finally, this easy-to-implement method can be considered to be a new tool for the in-plane thermal characterization of materials. It should be noted that when the materials become too conductive, the estimation method still remains valid. On the other hand, it is necessary to use heterodyne methods for the acquisition. ${ }^{20}$ In the future, polynomial development can be considered at higher orders for the detection of cracks perpendicular or parallel to the observation surface, mapping of thermophysical properties, and research of a small anomaly over a large area by using the pulsed flying spot technique.

\section{ACKNOWLEDGMENTS}

This work was supported by "Projet Région Aquitaine" and "Epsilon-Alcen" Industrial Groups.

${ }^{1}$ J. Krapez, L. Legrandjacques, F. Lepoutre, and D. Balageas, "Optimization of the photothermal camera for crack detection," in Proceedings of QIRT98 Conference (Seminar Eurotherm No 60), edited by D. Balageas, G. Busse, and G. M. Carlomagno (Akademickie Centrum Graficzno-Marketingowe Lodart SA, Ldz, Poland, 1998), Vol. 25, pp. 305-310; See www.qirt.org/dynamique/index.php?idD=55 for A QIRT Open Archives, Paper No. QIRT 1998-048.

${ }^{2}$ C. Gruss and D. Balageas, "Theoretical and experimental applications of the flying spot camera," in Proceedings of QIRT 92 Conference (Seminar Eurotherm No 27), edited by D. Balageas, G. Busse, and G. M. Carlomagno (Editions Europennes Thermique et Industrie, Paris, 1992), pp. 19-24; See www.qirt.org/dynamique/index.php?idD=55 for QIRT Open Archives, Paper No. QIRT 1992-004.

${ }^{3}$ Y. Wang, P. Kuo, L. Favro, and R. Thomas, "A novel flying-spot infrared camera for imaging very fast thermal-wave phenomena," in Photoacoustic and Photothermal Phenomena II, Springer Series in Optical Sciences (Springer, 1990), pp. 24-26.

${ }^{4}$ T. Li, D. P. Almond, and D. A. S. Rees, "Crack imaging by scanning pulsed laser spot thermography,” NDT \& E Int. 44(2), 216-225 (2011).

${ }^{5}$ J. C. Krapez, "Spatial resolution of the flying spot camera with respect to cracks and optical variations," in Proceedings of the 10th International Conference on Photoacoustic and Photothermal Phenomena (1999), Vol. 463 , pp. $377-379$.
${ }^{6} \mathrm{~S}$. Shepard and M. Frendberg, "Thermographic detection and characterization of flaws in composite materials," Mater. Eval. 72(7), 928 (2014).

${ }^{7}$ I. Philippi, J., Batsale, D. Maillet, and A. Degiovanni, "Measurement of thermal diffusivities through processing of infrared images," Rev. Sci. Instrum. 66(1), 182-192 (1995).

${ }^{8}$ J. C. Krapez, L. Spagnolo, M. Frieß, H. P. Maier, and G. Neuer, "Measurement of in-plane diffusivity in non-homogeneous slabs by applying flash thermography," Int. J. Thermal Sci. 43(10), 967-977 (2004).

${ }^{9}$ M. Bamford, M. Florian, G. L. Vignoles, J. C. Batsale, C. A. A. Cairo, and L. Maillé, "Global and local characterization of the thermal diffusivities of sic f/sic composites with infrared thermogra- phy and flash method," Compos. Sci. Technol. 69(7), 1131-1141 (2009).

${ }^{10}$ F. Cernuschi, A. Russo, L. Lorenzoni, and A. Figari, "In-plane thermal diffusivity evaluation by infrared thermography," Rev. Sci. Instrum. 72(10), 3988-3995 (2001).

${ }^{11} \mathrm{P}$. Bison, F. Cernuschi, and S. Capelli, "A thermographic technique for the simultaneous estimation of in-plane and in-depth thermal diffusivities of tbcs," Surf. Coat. Technol. 205(10), 3128-3133 (2011).

${ }^{12}$ H. S. Carslaw and J. C. Jaeger, Conduction of Heat in Solids, 2nd ed. (Clarendon Press, Oxford, 1959).

${ }^{13} \mathrm{M}$. Bamford and J. C. Batsale, "Analytical singular value decomposition of infrared image sequences: Microcrack detection on ceramic composites under mechanical stresses," C. R. Méc. 336(5), 440-447 (2008).

${ }^{14}$ C. Pradere, J. Morikawa, J. Toutain, J. C. Batsale, E. Hayakawa, and T. Hashimoto, "Microscale thermography of freezing biological cells in view of cryopreservation," Quant. Infrared Thermogr. J. 6(1), 37-61 (2009).

${ }^{15}$ J. C. Batsale and C. Pradere, "Infrared image processing devoted to thermal non-contact characterization-applications to non-destructive evaluation, microfluidics and $2 \mathrm{~d}$ source term distribution for multispectral tomography," J. Phys.: Conf. Ser. 655(1), 012002 (2015).

${ }^{16}$ A. L. Edwards, "A compilation of thermal property data for computer heat-conduction calculations," Report No. UCRL-50589, University of California Lawrence Radiation Laboratory, 1969.

${ }^{17}$ N. W. Pech-May, A. Mendioroz, and A. Salazar, "Simultaneous measurement of the in-plane and in-depth thermal diffusivity of solids using pulsed infrared thermography with focused illumination," NDT \& E Int. 77, 28-34 (2016).

${ }^{18}$ A. Degiovanni, "Correction de longueur d'impulsion pour la mesure de la diffusivité thermique par méthode flash," Int. J. Heat Mass Transfer 30(10), 2199-2200 (1987).

${ }^{19}$ M. Thomas, N. Boyard, N. Lefèvre, Y. Jarny, and D. Delaunay, "An experimental device for the simultaneous estimation of the thermal conductivity 3-D tensor and the specific heat of orthotropic composite materials,” Int. J. Heat Mass Transfer 53(23), 5487-5498 (2010).

${ }^{20}$ C. Pradere, L. Clerjaud, J. C. Batsale, and S. Dilhaire, "High speed heterodyne infrared thermography applied to thermal diffusivity identification," Rev. Sci. Instrum. 82, 054901 (2011), ISSN: 0034-6748. 